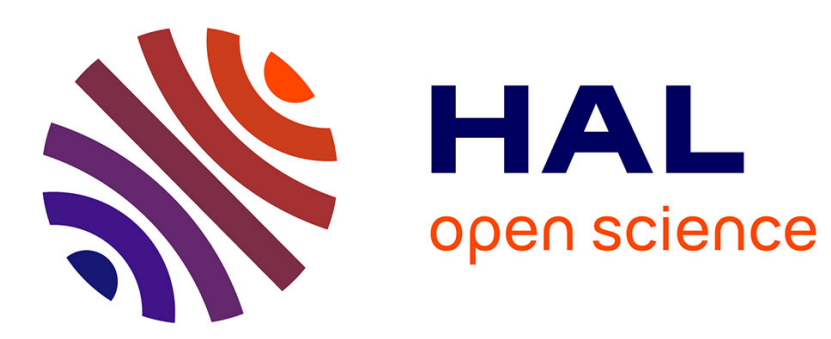

\title{
Ductile damage of porous materials with two populations of voids
}

Pierre-Guy Vincent, Yann Monerie, Pierre Suquet

\section{To cite this version:}

Pierre-Guy Vincent, Yann Monerie, Pierre Suquet. Ductile damage of porous materials with two populations of voids. Comptes Rendus Mécanique, 2008, 336 (1-2), pp.245-259. 10.1016/j.crme.2007.11.017 . hal-00214157

\section{HAL Id: hal-00214157 \\ https://hal.science/hal-00214157}

Submitted on 23 Jan 2008

HAL is a multi-disciplinary open access archive for the deposit and dissemination of scientific research documents, whether they are published or not. The documents may come from teaching and research institutions in France or abroad, or from public or private research centers.
L'archive ouverte pluridisciplinaire HAL, est destinée au dépôt et à la diffusion de documents scientifiques de niveau recherche, publiés ou non, émanant des établissements d'enseignement et de recherche français ou étrangers, des laboratoires publics ou privés. 


\title{
Ductile damage of porous materials with two populations of voids
}

\author{
Pierre-Guy Vincent ${ }^{\mathrm{a}}$, Yann Monerie ${ }^{\mathrm{a}}$, Pierre Suquet ${ }^{\mathrm{b}, 1}$ \\ a Institut de Radioprotection et de Sûreté Nucléaire, B.P. 3, 13115 Saint-Paul-lez-Durance Cedex France \\ ${ }^{\mathrm{b}}$ Laboratoire de Mécanique et d'Acoustique, 31, Chemin Joseph Aiguier, 13402 Marseille Cedex 20, France \\ Received *****; accepted after revision $* * * * * *$ \\ Presented by $* * * * * *$
}

\begin{abstract}
This study is devoted to the modelling of ductile damage in uranium dioxide. This polycrystalline material contains two populations of voids of well separated size. The problem addressed here is the prediction of the effective flow surface of a Gurson material containing randomly oriented oblate voids. The case of spherical voids is considered first and the variational approach of Gurson is generalized by adding a compressible component to his original velocity field. The case of aligned oblate voids is then considered and a suitable generalization of a velocity field due to Gologanu et al [6] is proposed. The extension to randomly oriented voids is achieved by averaging over all orientations. In each case, rigorous upper bounds and approximate estimates are derived and compared (in the case of spherical voids) with Finite Element simulations.
\end{abstract}

To cite this article: P.-G. Vincent et al., C. R. Mecanique ***** (2007).

\section{Résumé}

Endommagement ductile de matériaux poreux contenant deux populations de cavités. Cette note est consacrée à l'étude de l'endommagement ductile dans l'oxyde d'uranium. Ce matériau polycristallin présente deux familles de cavités de taille très différente. Le problème est abordé ici comme la recherche d'un critère de plasticité pour un matériau de Gurson contenant des cavités ellipsoïdales aplaties distribuées aléatoirement. Dans un premier temps, le cas des cavités sphériques est examiné. La démarche variationnelle de Gurson est reprise en ajoutant une composante compressible au champ de vitesse de Gurson. Cette démarche est ensuite étendue aux ellipsoïdes aplatis et alignés en reprenant et en complétant un champ de vitesse proposé par Gologanu et al [6]. Enfin l'extension aux ellipsoïdes orientés aléatoirement se fait par une prise de moyenne appropriée sur les orientations. Dans chaque cas, des bornes supérieures rigoureuses ainsi que des estimations sont proposées et comparées (seulement dans le cas des cavités sphériques) avec des calculs par éléments finis. Pour citer cet article : P.-G. Vincent et al., C. R. Mecanique ***** (2007).

Key words: Damage; Plasticity; Micromechanics

Mots-clés : Endommagement; Plasticité ; Micromécanique 


\section{Introduction}

The present work is devoted to the mechanical behavior of uranium dioxide $\left(\mathrm{UO}_{2}\right)$ studied by the French "Institut de Radioprotection et de Sûreté Nucléaire" (IRSN) to assess the safety of fuel rods under accident conditions. $\mathrm{UO}_{2}$ is a polycrystalline material which, when highly irradiated, exhibits a microstructure with two populations of voids of rather different sizes and shapes as schematically depicted in Figure 1 (micrographs of the actual material can be found in [3]). The smallest voids are intragranular and almost spherical in shape. The largest voids are elongated and located at grain boundaries (see Figure 1). At high temperature the ceramic matrix is ductile and under accident conditions the increase in pressure in the voids can cause the voids to grow and to coalesce, eventually leading to the formation of a macroscopic crack. This process is very similar to that of ductile rupture in metals (Leblond [9]) and the present study is an attempt to analyze damage evolution in $\mathrm{UO}_{2}$ by similar means.

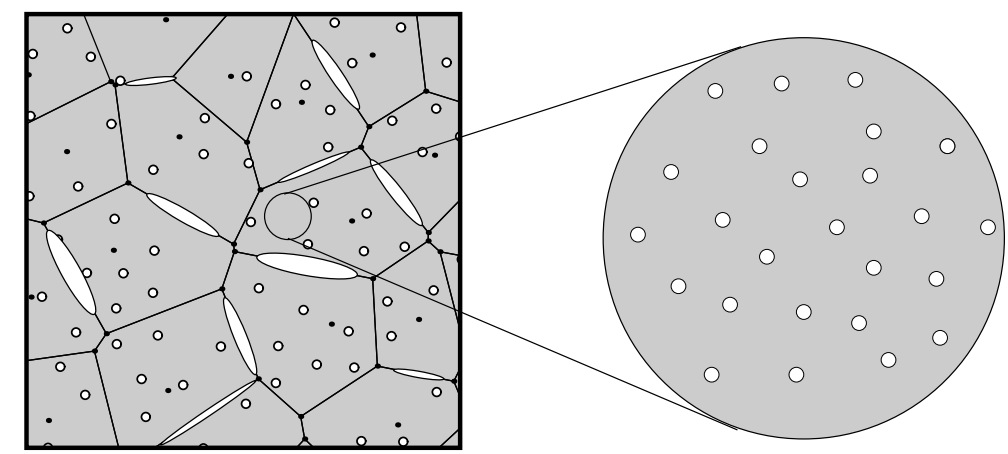

Figure 1. Left: polycrystalline structure of $\mathrm{UO}_{2}$ showing intergranular lenticular and intragranular voids. Right: close-up on the spherical intragranular voids.

Three different scales are relevant in this problem. The microscopic scale corresponds to the single grain level where the spherical pores can be distinguished (Figure 1 right). At the mesoscopic (or intermediate) scale (Figure 1 left) the smallest voids are "smeared out" and the different grains appear as different domains with different orientations of the same porous material. Then, at the macroscopic scale all voids are smeared out and the polycrystal is seen as a homogeneous material. The objective of this study is to derive an expression for the effective (macroscopic) yield surface of such bi-porous materials when the sound material at the microscopic scale is a ductile ideally plastic von Mises material (with yield stress $\left.\sigma_{0}\right)$.

Let $V$ denote a representative volume element of this material at the mesoscopic scale. $V$ consists of a domain $M$ occupied by the (porous) matrix (the smallest voids being smeared out), the remaining domain $V-M$ being occupied by the large voids. Two different measures of porosity corresponding to the different scales involved in the problem should be distinguished. At the mesoscopic scale (single grain scale) the volume fraction of the smallest voids is denoted by $f_{b}$. At the macroscopic scale (polycrystal) the volume fraction of the largest voids in $V$ is denoted by $f_{e}$. The total porosity $f$ is obtained by considering the volume fraction of the sound material and reads as $f=f_{e}+f_{b}-f_{e} f_{b}$.

At the mesoscopic scale the plastic flow of the porous matrix, with porosity $f_{b}$, is assumed to be governed by the Gurson-Needleman-Tvergaard (GTN) yield function:

\footnotetext{
Email addresses: pierre-guy.vincent@irsn.fr (Pierre-Guy Vincent), yann.monerie@irsn.fr (Yann Monerie), suquet@lma.cnrs-mrs.fr (Pierre Suquet).

1 Corresponding author.
} 


$$
q_{3}\left(\frac{\sigma_{e q}}{\sigma_{0}}\right)^{2}+2 q_{1} f_{b} \cosh \left(\frac{3}{2} \frac{\sigma_{m}}{\sigma_{0}}\right)-1-\left(q_{1} f_{b}\right)^{2}=0,
$$

where $\sigma_{m}$ and $\sigma_{e q}$ denote the usual hydrostatic stress and equivalent stress. The original Gurson yield function corresponds to $q_{1}=q_{3}=1$ [7]. The $q_{i}$ 's are adjustable parameters introduced by Tvergaard and Needleman (see for instance [18]) to fit the yield function (1) with numerical simulations. The most popular choices in the literature are $q_{1}$ ranging from 1.25 to 1.5 and $q_{3}=1$, but Leblond et al [8] have introduced $q_{3}=1+2 / 3 f_{b}$ to satisfy a nonlinear variational upper bound for porous ductile materials.

In this study an expression for the effective yield surface of porous materials, consisting of a GTN matrix and containing ellipsoidal cavities, is derived. A variational approach is adopted to characterize the effective dissipation potential of the (bi-)porous material. Upper bounds for the effective strain-rate potential of the representative volume element can be obtained from the variational principle:

$$
\Phi(\dot{\boldsymbol{E}})=\inf _{v \in \mathcal{K}(\dot{\boldsymbol{E}})} \frac{1}{|V|} \int_{M} \varphi(\varepsilon(\boldsymbol{v})) d V \quad \text { with } \mathcal{K}(\dot{\boldsymbol{E}}) \equiv\{\boldsymbol{v} \mid \boldsymbol{v}=\dot{\boldsymbol{E}} \cdot \boldsymbol{x} \text { on } \partial V\},
$$

where $\dot{\boldsymbol{E}}$ is the overall strain-rate and $\varphi$ is the matrix dissipation potential which will be specified in due time.

To make the problem more amenable to analytical calculations, the representative volume element $V$ under consideration is seen as an assemblage of self-similar hollow spheroids (axisymmetric ellipsoids) which are identical copies, after dilatation and rotation, of a unit hollow ellipsoid as shown in Figure 2. This unit hollow ellipsoid, denoted by $\Omega$, contains at its center an ellipsoidal cavity $\omega$. The outer and inner surfaces are confocal ellipsoids.

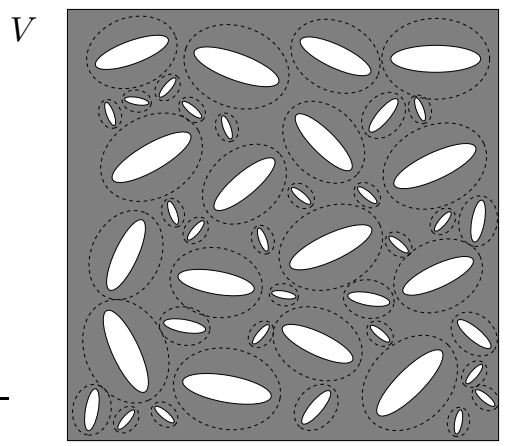

(a)

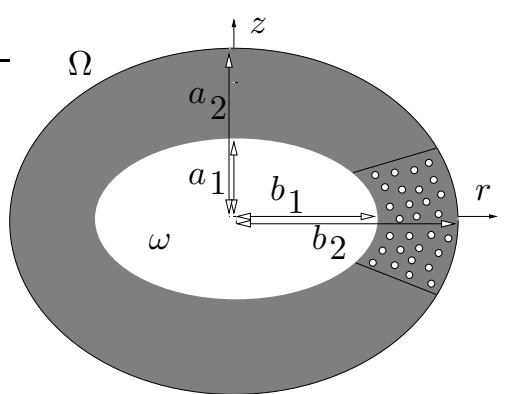

(b)

Figure 2. (a) Assemblage of ellipsoids. (b) Single hollow ellipsoid.

This paper is organized as follows. Section 2 is devoted to the case where the large voids are spherical. Rigorous upper bounds and estimates are derived. In particular an estimate in the form of a generalized Gurson's yield function is proposed in equation (14). The resulting predictions are compared with Finite Element simulations. The approximation which is made in the velocity field consisting in a uniform dilatation in the matrix, does not affect significantly the response of such a bi-porous material. The case of oblate ellipsoidal voids in a Gurson matrix is addressed in section 3. The velocity field of Gologanu et al [6] is recalled first and used in section 3.2 to derive an estimate for a single hollow ellipsoid. The case of randomly oriented oblate ellipsoidal voids is considered in section 3.3 where an upper bound for the effective potential is derived. Finally an estimate of the yield function for a Gurson material containing randomly oriented ellipsoidal voids is derived in section 3.4. 


\section{Spherical voids}

Let us consider first the case where the largest pores are spherical. The unit volume element is a hollow sphere with internal and external radii $a_{1}$ and $a_{2}$ respectively.

The problem of a hollow sphere made of a Gurson matrix under hydrostatic loading has been investigated analytically by Perrin and Leblond $[13,14,15]$, but remains widely open for more general loading conditions. A variational approach is followed here.

\subsection{Incompressible matrix: single hollow sphere and assemblage}

Let us briefly recall the derivation (due to Perrin [14]) of Gurson's yield function when there is no small voids $\left(f_{b}=0\right)$. In this case the matrix potential reduces to $\varphi(\dot{\varepsilon})=\sigma_{0} \dot{\varepsilon}_{e q}$. Using the variational principle (2) with a velocity field which combines the exact velocity under hydrostatic loading and a homogeneous and purely deviatoric deformation field, namely:

$$
\boldsymbol{v}^{G}(\dot{\boldsymbol{E}})=\dot{E}_{m} \frac{a_{2}^{3}}{r^{2}} \boldsymbol{e}_{r}+\boldsymbol{D} \cdot \boldsymbol{x}, \quad \boldsymbol{D}=\dot{\boldsymbol{E}}-\dot{E}_{m} \boldsymbol{i},
$$

where $\boldsymbol{i}$ is the second-order identity tensor, the effective potential for the unit-volume element is found to be bounded from above by:

$$
\Phi(\dot{\boldsymbol{E}}) \leq \frac{1}{|\Omega|} \int_{\Omega-\omega} \varphi\left(\varepsilon\left(\boldsymbol{v}^{G}\right)\right) d \Omega \leq \frac{\sigma_{0}}{|\Omega|} \int_{r=a_{1}}^{r=a_{2}} S(r) \sqrt{\frac{1}{S(r)} \int_{\phi=0}^{2 \pi} \int_{\theta=0}^{\pi} \dot{\varepsilon}_{e q}^{2} d S} d r
$$

where $\dot{\boldsymbol{\varepsilon}}=\boldsymbol{\varepsilon}\left(\boldsymbol{v}^{G}\right), S(r)=4 \pi r^{2}$ and $d S=r^{2} \sin \theta d \theta d \phi$ (spherical coordinates $\left.r, \theta, \phi\right)$. After a few algebraic manipulations, one finally obtains [2]:

$$
\Phi(\dot{\boldsymbol{E}}) \leq \Phi^{G}(\dot{\boldsymbol{E}})=\sigma_{0} \int_{f_{e}}^{1} \sqrt{\frac{4 \dot{E}_{m}^{2}}{y^{2}}+\dot{E}_{e q}^{2}} d y,
$$

where $\Phi^{G}$ is the dissipation potential associated with the yield surface (1) with $q_{1}=q_{3}=1$ and $f_{e}$ instead of $f_{b}$.

\subsection{Compressible GTN matrix: upper bound for the single hollow sphere and the assemblage}

When the matrix obeys the GTN yield function (1), the dissipation potential entering the variational principle (2) reads:

$$
\varphi(\dot{\varepsilon})=\sigma_{0} \int_{q_{1} f_{b}}^{1} \sqrt{\frac{4 \dot{\varepsilon}_{m}^{2}}{y^{2}}+\frac{\dot{\varepsilon}_{e q}^{2}}{q_{3}}} d y .
$$

Use is made of a compressible velocity field in the form

$$
\boldsymbol{v}=A \boldsymbol{x}+\boldsymbol{v}^{G}(\dot{\boldsymbol{E}}-A \boldsymbol{i}) .
$$

The velocity field (6) has a uniform dilatation-rate in the matrix $\dot{\varepsilon}_{m}=A$. The following upper bound for the effective potential is then obtained from the variational principle:

$$
\Phi(\dot{\boldsymbol{E}}) \leq \inf _{A} \frac{1}{|\Omega|} \int_{\Omega-\omega} \sigma_{0}\left(\int_{q_{1} f_{b}}^{1} \sqrt{\frac{4 A^{2}}{y^{2}}+\frac{\varepsilon_{e q}^{2}\left(v^{G}\right)}{q_{3}}} d y\right) d \Omega .
$$


Applying the Cauchy-Schwarz inequality on each concentric sphere, one obtains:

$$
\Phi(\dot{\boldsymbol{E}}) \leq \inf _{A} \frac{\sigma_{0}}{|\Omega|} \int_{q_{1} f_{b}}^{1} \int_{r=a}^{r=b} S(r) \sqrt{\frac{4 A^{2}}{y^{2}}+\frac{1}{S(r)} \int_{\phi=0}^{2 \pi} \int_{\theta=0}^{\pi} \frac{\varepsilon_{e q}^{2}\left(v^{G}\right)}{q_{3}} d S} d r d y,
$$

and finally

$$
\Phi(\dot{\boldsymbol{E}}) \leq \inf _{A} \sigma_{0} \int_{q_{1} f_{b}}^{1} \int_{f_{e}}^{1} \sqrt{\frac{4 A^{2}}{y^{2}}+\frac{4}{q_{3}} \frac{\left(\dot{E}_{m}-A\right)^{2}}{z^{2}}+\frac{\dot{E}_{e q}^{2}}{q_{3}}} d z d y .
$$

The right-hand side of (8) provides a rigorous upper-bound to the effective potential of a unit-volume element which can be extended by a classical argument into an upper bound for the potential of any composite sphere assemblage.

To the best of our knowledge, the minimization with respect to $A$ in the upper bound (8) cannot be performed in closed form. An approximation preserving the upper bound character of (8) can be obtained by making the particular choice:

$$
A=\bar{A} \equiv \frac{q_{1} f_{b}}{f_{e}+q_{1} f_{b}} \dot{E}_{m} .
$$

It should be noted that $\bar{A}$ is the exact minimizer of (8) in several particular cases. First, when $f_{b}$ (resp. $f_{e}$ ) vanishes, it is easy to see that $A=0$ (resp. $A=\dot{E}_{m}$ ) is solution of the infimum problem in (8). Similarly, when $q_{3}=1$ and $q_{1} f_{b}=f_{e}, A=1 / 2 \dot{E}_{m}$ is the solution of (8). Specifying $A=\bar{A}$ in (8) gives the following upper bound:

$$
\Phi(\dot{E}) \leq \sigma_{0} \int_{q_{1} f_{b}}^{1} \int_{f_{e}}^{1} \sqrt{\frac{4 q_{1}^{2} f_{b}^{2}}{\left(f_{e}+q_{1} f_{b}\right)^{2}} \frac{\dot{E}_{m}^{2}}{y^{2}}+\frac{4 f_{e}^{2}}{q_{3}\left(f_{e}+q_{1} f_{b}\right)^{2}} \frac{\dot{E}_{m}^{2}}{z^{2}}+\frac{\dot{E}_{e q}^{2}}{q_{3}}} d z d y .
$$

Remark: In the practical application that we have in mind, the two populations of voids are subjected to internal pressures, $p_{b}$ in the small voids and $p_{e}$ in the largest voids. The variational principles (2) and (8) must be modified accordingly by making the following replacements:

$$
\left.\begin{array}{rl}
\varphi(\dot{\boldsymbol{\varepsilon}}) & \rightarrow \varphi_{b}(\dot{\boldsymbol{\varepsilon}})=\varphi(\dot{\boldsymbol{\varepsilon}})-3 p_{b} \dot{\varepsilon}_{m}, \\
\frac{1}{|V|} \int_{M} \varphi(\varepsilon(\boldsymbol{v})) d V & \rightarrow \frac{1}{|V|}\left[\int_{M} \varphi_{b}(\varepsilon(\boldsymbol{v})) d V-3 p_{e} \int_{V-M} \dot{\varepsilon}_{m} d V\right], \\
\Phi(\dot{E}) & \rightarrow \Phi(\dot{E})-3 p_{e} \dot{E}_{m}+3\left(1-f_{e}\right)\left(p_{e}-p_{b}\right) A .
\end{array}\right\}
$$

Then, a minimization with respect to $A$ has to be performed on the modified variational principle (8). It should be emphasized that the approximate expression (9) does not hold true when the internal pressures $p_{e}$ and $p_{b}$ are different. In the interest of space, the influence of the internal pressures will not be studied here and is left for separate work.

\subsection{Compressible GTN matrix: Gurson-like estimate for the single hollow sphere and the assemblage}

The double integral in (8) and (10) can be expressed in closed form by means of the relations given in Appendix A. However the resulting expression is too complicated to find an explicit equation for the yield surface corresponding to the dissipation potential (10). Instead, an approximate expression can be proposed. For this purpose it is noted that, when specialized to purely deviatoric and purely hydrostatic conditions, the upper bound (10) gives two particular stress states located on the effective yield surface of the material:

$$
\Sigma_{m}=0, \quad \Sigma_{e q}=\widetilde{\sigma}_{0} \quad \text { with } \quad \widetilde{\sigma}_{0}=\sigma_{0}\left(1-f_{b}\right)\left(1-f_{e}\right) / \sqrt{q_{3}}
$$


and

$$
\Sigma_{m}=\widetilde{p}, \quad \Sigma_{e q}=0 \quad \text { with } \quad \widetilde{p}=\frac{1}{3} \sigma_{0} \frac{2}{\sqrt{q_{3}}\left(f_{b}+f_{e}\right)}\left(C_{1}+\ln \left(C_{2}\right)\right),
$$

where

$$
\begin{gathered}
B_{1}=\sqrt{f_{e}^{2}+f_{b}^{2} q_{3}}, \quad B_{2}=f_{b} \sqrt{f_{e}^{2}+q_{3}}, \quad B_{3}=f_{e} \sqrt{1+f_{b}^{2} q_{3}}, \\
B_{4}=f_{b} f_{e} \sqrt{1+q_{3}}, \quad C_{1}=2\left(B_{1}-B_{2}-B_{3}+B_{4}\right), \\
C_{2}=f_{b}^{f_{b}\left(1-f_{e}\right) \sqrt{q_{3}}} f_{e}^{\left(1-f_{b}\right) f_{e}}\left(\frac{B_{1}-f_{e}}{B_{3}-f_{e}}\right)^{f_{e}}\left(\frac{B_{4}-f_{b} f_{e}}{B_{2}-f_{b} f_{e}}\right)^{f_{b} f_{e}}\left(\frac{B_{1}-f_{b} \sqrt{q_{3}}}{B_{2}-f_{b} \sqrt{q_{3}}}\right)^{f_{b} \sqrt{q_{3}}}\left(\frac{B_{4}-f_{b} f_{e} \sqrt{q_{3}}}{B_{3}-f_{b} f_{e} \sqrt{q_{3}}}\right)^{f_{b} f_{e} \sqrt{q_{3}}} .
\end{gathered}
$$

The second approximation which is introduced here consists in enforcing the form (1) as an approximation (hopefully accurate) for the effective yield surface of the bi-porous material which reads as:

$$
\frac{1}{\beta}\left(\frac{\Sigma_{e q}}{\sigma_{0}}\right)^{2}+\frac{1}{\alpha} \cosh \left(\frac{3}{2} \frac{\Sigma_{m}}{\sigma_{0}}\right)-1=0, \quad \text { with } \alpha \equiv \cosh \left(\frac{3}{2} \frac{\widetilde{p}}{\sigma_{0}}\right) \text { and } \beta \equiv \frac{\alpha}{\alpha-1}\left(\frac{\widetilde{\sigma}_{0}}{\sigma_{0}}\right)^{2} .
$$

This yield function can be further refined by improving the prediction (12) for purely deviatoric stress. This is done by means of a variational argument close to that developed by Suquet [17].

\subsection{Variational bound and refined estimate}

Coming back to the variational characterization (2), the effective potential $\Phi$ can be written as:

$$
\Phi(\dot{\boldsymbol{E}}) \leq \inf _{\boldsymbol{v} \in \mathcal{K}(\dot{\boldsymbol{E}})} \frac{\sigma_{0}}{|V|} \int_{M} \int_{q_{1} f_{b}}^{1} \sqrt{\frac{4 \dot{\varepsilon}_{m}^{2}}{y^{2}}+\frac{\dot{\varepsilon}_{e q}^{2}}{q_{3}}} d y d V .
$$

The Cauchy-Schwarz inequality is applied first to the integral on $y$ :

$$
\Phi(\dot{\boldsymbol{E}}) \leq \frac{\sigma_{0} \sqrt{1-q_{1} f_{b}}}{|V|} \inf _{\boldsymbol{v} \in \mathcal{K}(\dot{\boldsymbol{E}})} \int_{M}\left(\frac{4\left(1-q_{1} f_{b}\right)}{q_{1} f_{b}} \dot{\varepsilon}_{m}^{2}+\frac{\left(1-q_{1} f_{b}\right)}{q_{3}} \dot{\varepsilon}_{e q}^{2}\right)^{1 / 2} d V
$$

and then to the integral on $V$ :

$$
\Phi(\dot{\boldsymbol{E}}) \leq \sigma_{0} \sqrt{1-q_{1} f_{b}} \sqrt{1-f_{e}}\left[\inf _{\boldsymbol{v} \in \mathcal{K}(\dot{\boldsymbol{E}})} \frac{1}{|V|} \int_{M}\left(\frac{9}{2} k_{1} \dot{\varepsilon}_{m}^{2}+\frac{3}{2} \mu_{1} \dot{\varepsilon}_{e q}^{2}\right) d V\right]^{1 / 2}
$$

where

$$
k_{1}=\frac{8\left(1-q_{1} f_{b}\right)}{9 q_{1} f_{b}}, \quad \mu_{1}=\frac{2\left(1-q_{1} f_{b}\right)}{3 q_{3}} .
$$

Therefore when the composite has overall isotropy:

$$
\Phi(\dot{\boldsymbol{E}}) \leq \sigma_{0} \sqrt{1-q_{1} f_{b}} \sqrt{1-f_{e}}\left(\frac{9}{2} \widetilde{k}_{1} \dot{E}_{m}^{2}+\frac{3}{2} \widetilde{\mu}_{1} \dot{E}_{e q}^{2}\right)^{1 / 2},
$$

where $\widetilde{k}_{1}$ and $\widetilde{\mu}_{1}$ denote the effective bulk and shear moduli of a linear porous material with bulk and shear moduli $k_{1}$ and $\mu_{1}$ respectively.

As is now well-recognized the bounds obtained by (most) variational techniques are rather poor for hydrostatic loadings but sharp under purely deviatoric loadings. Therefore a sharper estimate for the flow surface of the bi-porous material can be obtained by considering the yield function (14) with (13) and:

$$
\Sigma_{m}=0, \quad \Sigma_{e q}=\widetilde{\sigma}_{0} \quad \widetilde{\sigma}_{0}=\sigma_{0} \sqrt{\left(1-q_{1} f_{b}\right)\left(1-f_{e}\right)} \sqrt{\frac{3}{2} \widetilde{\mu}_{1}} \dot{E}_{e q} .
$$


In the absence of further information on the composite microstructure besides overall isotropy, the sharpest bounds on the effective moduli $\widetilde{k}_{1}$ and $\widetilde{\mu}_{1}$ are the Hashin-Shtrikman bounds (only the upper bound is relevant here):

$$
\widetilde{k}_{1} \leq \frac{4}{3} \mu_{1} \frac{1-f_{e}}{f_{e}+\frac{4 \mu_{1}}{3 k_{1}}}, \quad \widetilde{\mu}_{1} \leq \mu_{1} \frac{1-f_{e}}{1+6 f_{e} \frac{k_{1}+2 \mu_{1}}{9 k_{1}+8 \mu_{1}}},
$$

and the following upper bound for $\Phi$ is obtained:

$$
\Phi(\dot{\boldsymbol{E}}) \leq \sigma_{0} \frac{\left(1-q_{1} f_{b}\right)\left(1-f_{e}\right)}{\sqrt{q_{3}}}\left(\frac{4 \dot{E}_{m}^{2}}{f_{e}+\frac{q_{1} f_{b}}{q_{3}}}+\frac{\dot{E}_{e q}^{2}}{1+f_{e} \frac{2 q_{3}+3 q_{1} f_{b}}{3 q_{3}+2 q_{1} f_{b}}}\right)^{1 / 2},
$$

The corresponding point under purely deviatoric loading reads as :

$$
\Sigma_{m}=0, \quad \Sigma_{e q}=\tilde{\sigma}_{0}, \quad \tilde{\sigma}_{0}=\sigma_{0} \frac{\left(1-q_{1} f_{b}\right)\left(1-f_{e}\right)}{\sqrt{q_{3}}} \sqrt{\frac{1}{1+f_{e} \frac{2 q_{3}+3 q_{1} f_{b}}{3 q_{3}+2 q_{1} f_{b}}}} .
$$

The resulting estimate (14) is a rigorous upper bound for purely hydrostatic or purely deviatoric overall stress. As can be seen in Figure 3 this estimate remains an upper bound for intermediate stress triaxiality ratios.

\subsection{Comparison with Finite Element simulations}

To assess the accuracy of the upper bound (8) and of the estimate (14) with (13) and (17), the predictions of these models for the effective yield surface of the bi-porous material are compared in Figure 3 with full-field numerical simulations obtained with the Finite Element Method. Attention is limited in the numerical simulations to axisymmetric deformations, one quarter of a hollow sphere is meshed and boundary conditions corresponding to uniform deformations are imposed on the outer boundary of the hollow sphere. The matrix is a Gurson material with $q_{1}=q_{3}=1$. Different radial paths in the space of macroscopic stress are followed (a stress-driven method similar to that described in Michel et al [10] is used).

As can be seen from Figure 3, the upper bound (8) and the estimate (14) are in good agreement. The exact result of Perrin [14] for hydrostatic loading has been evaluated numerically and is indicated as a vertical line in this figure. Both the upper bound (8) and the estimate are in very good agreement with this exact result. The results of the FEM simulations are shown as full squares. As expected they lie inside the prediction of the upper bound (8), with an excellent agreement under hydrostatic stress and a good agreement under purely deviatoric stress. The quality of the match between the analytical equation (14) with (13) and (17) and the FEM simulations is excellent for purely hydrostatic or deviatoric stress and good at all other stress triaxiality ratios. The Gurson flow surface with total porosity $f=f_{e}+f_{b}-f_{e} f_{b}$ is also shown as dashed lines. The motivation for considering the total porosity is that Perrin and Leblond $[13,15]$ observed for $f_{b}$ and $f_{e}$ small enough (which is the case in practice), a good agreement between their exact result under hydrostatic loading and the corresponding prediction of the Gurson yield function with total porosity $f$. The present model $(8)$ coincides with the Gurson model with porosity $f$ under purely deviatoric loading conditions but differs from it under hydrostatic loading. Overall, the Gurson model with total porosity $f$ is in reasonable agreement with the other models. However it seems to slightly overestimate the effective carrying capacity of the bi-porous material and its agreement with the FEM simulations is less satisfactory than that of the other, more refined, models. 


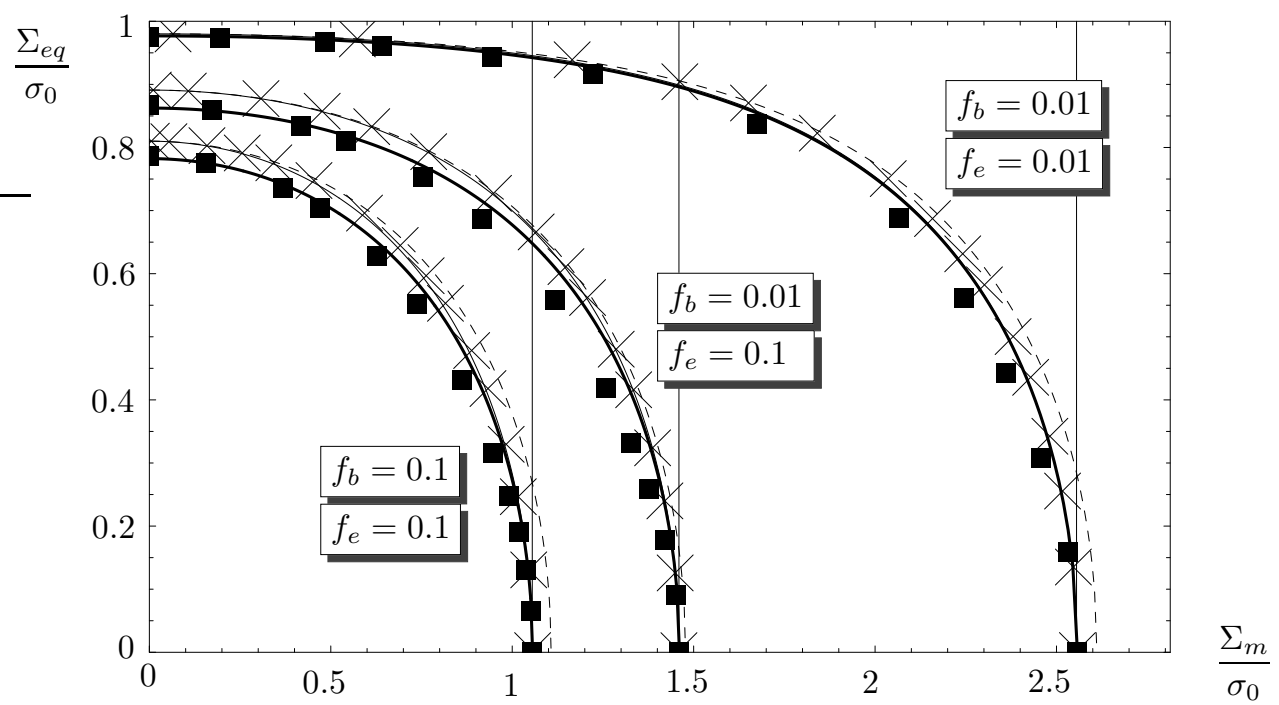

Figure 3. Effective yield surface for a porous material with two populations of voids for different void volume fractions $f_{b}$ (small voids) and $f_{e}$ (large voids). Upper bound (8) (crosses), estimate (14) with (13) and (12) (black solid line), estimate (14) with (13) and (17) (thick black solid line), FEM simulations (full squares), hydrostatic point of [14] (vertical line), Gurson yield function with total porosity $f$ (dashed line).

\subsection{Local dilatation rate and evolution of the damage parameters}

The mass balance equations written at the mesoscopic and macroscopic scales respectively yield:

$$
\dot{f}_{b}=3\left(1-f_{b}\right) \dot{\varepsilon}_{m}, \quad \dot{f}=3(1-f) \dot{E}_{m},
$$

which, for the present model where $\dot{\varepsilon}_{m}=A$, yields by virtue of the relations between $f, f_{e}$ and $f_{b}$ :

$$
\dot{f}_{e}=3\left(1-f_{e}\right)\left(\dot{E}_{m}-A\right) \text {. }
$$

The first equation in (18) raises the question of the accuracy of the dilatation rate $\dot{\varepsilon}_{m}$ which is approximated by $A$ in the present approach.

The velocity field (6) corresponds to a uniform dilatation-rate throughout the matrix. This particular choice is legitimate since it yields an admissible velocity field, but can be questioned in view of the analytical result of Perrin and Leblond $[13,15]$ who showed that the dilatation-rate in a porous hollow sphere under hydrostatic loading is nonuniform. A comparison between the (uniform) dilatation rate predicted by the minimization of (8), the estimate (9) for this dilatation rate, the exact result of [14] and the prediction of the $N$-phase secant method (in the spirit of Bilger et al [1]) is shown in Figure 4 $\left(q_{1}=q_{3}=1\right)$.

Incorporating the exact result of [14] into a predictive scheme is not an easy task. One of the difficulties is that accounting for the nonuniformity of the dilatation-rate during an evolution process requests considering the secondary porosity $f_{b}$ (corresponding to the smaller voids) as a field rather than as a parameter $f_{b}$ uniform throughout the matrix. Keeping track of this field along the evolution of the porous material is a formidable task which is beyond the scope of this study. It is very likely that such porosity fluctuations play an important role in the coalescence process, but it is our belief that the part of the damage evolution corresponding to the cavity growth should be correctly approximated by the present model considering that the secondary porosity remains uniform. 

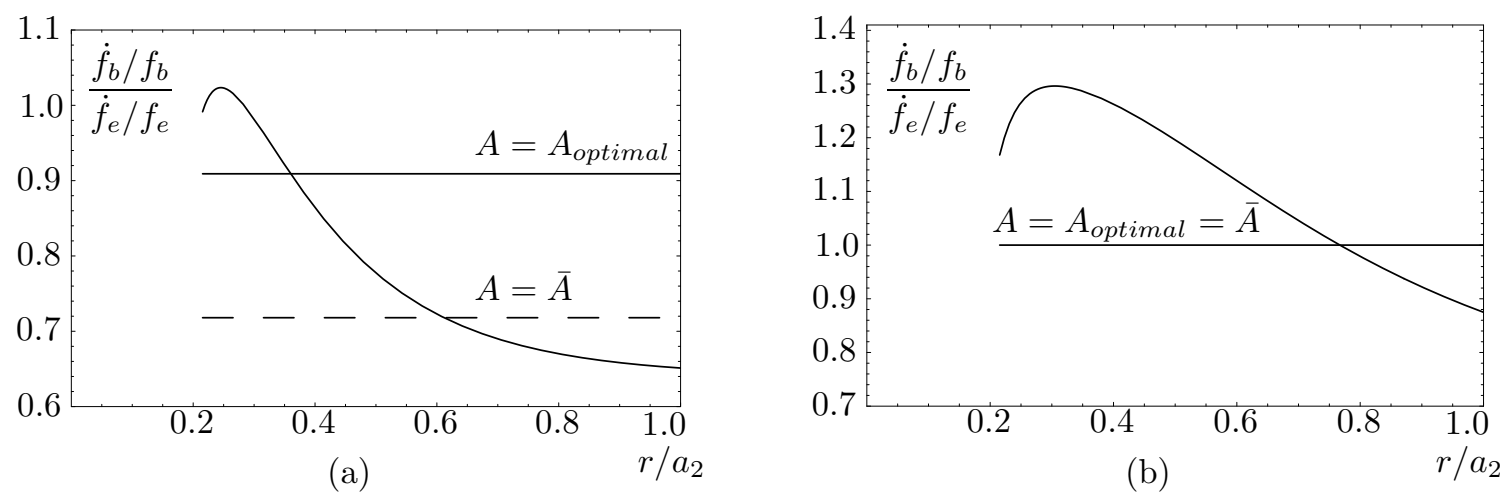

Figure 4. Hollow sphere under hydrostatic tension: relative rate-growth of the damage parameters. Exact result (Perrin [14], solid line; or equivalently $N$-phase secant method with $N \geq 100$ ), predictions based on the $A_{\text {optimal }}$ minimizing (8) (horizontal solid line) or on the approximation (9) (horizontal dashed line). (a) $f_{e}=1 \%, f_{b}=10 \%$. (b) $f_{e}=1 \%$, $f_{b}=1 \%$.

To check this assertion the response of a hollow sphere, consisting of a Gurson matrix $\left(q_{1}=q_{3}=1\right.$, porosity $f_{b}$ uniform at time $t=0$ in the matrix) containing a spherical central cavity, and subjected to hydrostatic strain-rate has been integrated in time. At each time step the change in volume of the central cavity and the change in volume of the secondary porosity are evaluated using the evolution equations (18). Different predictions are shown in Figure 5, the response of the hollow sphere as predicted by the $N$-phase modified secant method (with $N=101$ we expect these results to be almost exact under hydrostatic loading as it was the case for a von Mises matrix [1]), the predictions of the upper bound (8) and of the estimate (14). Surprizingly, all the predictions lie almost on top of each other, meaning that neglecting the spatial heterogeneity of the secondary porosity $f_{b}$ does not affect significantly the response of the porous material.

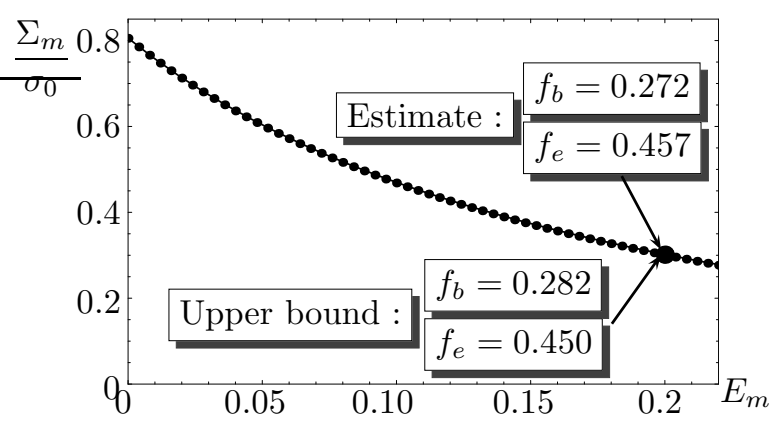

(a)

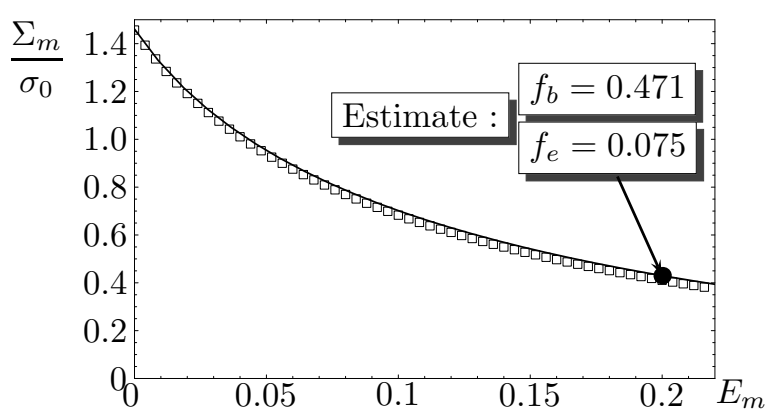

(b)

Figure 5. Macroscopic response of a porous hollow sphere under hydrostatic loading. Full circles : upper bound (10) ; Black Solid Line : estimate (14). White Squares: modified secant method (100 layers surrounding the cavity $\omega$; a local porosity $f_{b}^{i}$ is defined in each layer). (a) Initial porosities : $f_{b}=0.1, f_{e}=0.2$. (b) Initial porosities : $f_{b}=0.1, f_{e}=0.01$.

\section{Oblate ellipsoidal voids}

We now turn to the case where the large voids are oblate spheroids with random orientations. 


\subsection{Incompressible matrix: single hollow ellipsoid.}

We consider first the case where the matrix is an incompressible von Mises material (no small voids at the microscopic scale). To simplify things further, the volume element is assumed to be a single hollow ellipsoid. More specifically the outer and inner surfaces of this hollow domain are confocal ellipsoids and one can introduce a continuous family of ellipsoids parametrized by a dimensionless scalar parameter $\lambda$ such that the inner surface corresponds to $\lambda=\lambda_{1}$ and the outer surface corresponds to $\lambda=\lambda_{2}$. The axis of revolution of the two oblate ellipsoids is the $z$ axis (see Appendix B for more details).

The macroscopic strain-rate is split into an axisymmetric part and a non axisymmetric part $\dot{\boldsymbol{E}}^{N A}$ :

$$
\dot{\boldsymbol{E}}=\dot{\boldsymbol{E}}^{A}+\dot{\boldsymbol{E}}^{N A} \text { with } \dot{\boldsymbol{E}}^{A}=\frac{\dot{E}_{x x}+\dot{E}_{y y}}{2}\left(\boldsymbol{e}_{x} \otimes \boldsymbol{e}_{x}+\boldsymbol{e}_{y} \otimes \boldsymbol{e}_{y}\right)+\dot{E}_{z z} \boldsymbol{e}_{z} \otimes \boldsymbol{e}_{z}, \dot{\boldsymbol{E}}^{N A}=\dot{\boldsymbol{E}}-\dot{\boldsymbol{E}}^{A} .
$$

The velocity field in the matrix is chosen of the following form:

$$
\boldsymbol{v}(\boldsymbol{x})=\boldsymbol{v}^{G o}(\boldsymbol{x})+\boldsymbol{v}^{N A}(\boldsymbol{x}) \text { with } \boldsymbol{v}^{N A}(\boldsymbol{x})=\dot{\boldsymbol{E}}^{N A} \cdot \boldsymbol{x},
$$

where the velocity field $\boldsymbol{v}^{\text {Go }}$ (recalled in Appendix B) has been proposed by Gologanu et al [6] and satisfies $\boldsymbol{v}^{G o}=\dot{\boldsymbol{E}}^{A} \cdot \boldsymbol{x}$ on $\partial \Omega$. It follows from the variational property (2) that

$$
\Phi(\dot{\boldsymbol{E}}) \leq \frac{\sigma_{0}}{|\Omega|} \int_{\Omega-\omega} \dot{\varepsilon}_{e q} d \Omega \leq \sigma_{0} \int_{\lambda_{1}}^{\lambda_{2}} \sqrt{J(\lambda)} \sqrt{\int_{\phi=0}^{2 \pi} \int_{\beta=0}^{\pi} \dot{\varepsilon}_{e q}^{2} J_{\lambda \beta \phi} d \beta d \phi} d \lambda,
$$

with

and

$$
\dot{\varepsilon}=\varepsilon(\boldsymbol{v}), \quad \dot{\varepsilon}_{e q}^{2}=\left(\dot{\varepsilon}_{e q}^{G o}\right)^{2}+\left(\dot{E}_{e q}^{N A}\right)^{2}+\frac{4}{3} \dot{\varepsilon}^{G o}: \dot{\boldsymbol{E}}^{N A},
$$

$J_{\lambda \beta \phi}=\frac{b\left(a^{2} \sin ^{2} \beta+b^{2} \cos ^{2} \beta\right) \sin \beta}{|\Omega|}, \quad J(\lambda)=\int_{\phi=0}^{2 \pi} \int_{\beta=0}^{\pi} J_{\lambda \beta \phi} d \beta d \phi=\frac{4}{3} \frac{\pi b\left(2 a^{2}+b^{2}\right)}{|\Omega|}, \quad|\Omega|=\frac{4}{3} \pi a_{2} b_{2}^{2} \quad$.

It can be shown that the integral of the cross-term in the expression of $\dot{\varepsilon}_{e q}^{2}$ cancels out. After a few algebraic manipulations, this inequality simplifies into:

$$
\Phi(\dot{\boldsymbol{E}}) \leq \sigma_{0} \int_{\lambda_{1}}^{\lambda_{2}} \sqrt{J(\lambda)} \sqrt{\dot{\boldsymbol{E}}: \boldsymbol{Q}(\lambda): \dot{\boldsymbol{E}}} d \lambda
$$

where the detailed expression of the fourth-order tensor $\boldsymbol{Q}$ is given in the Appendix B.

\subsection{Compressible GTN matrix: single hollow ellipsoid.}

The matrix is now a GTN material whose dissipation potential $\varphi$ is given by (5). Following the same procedure as in section 2.2 , the trial velocity field in the matrix is the sum of the incompressible velocity field (20) and of a compressible contribution:

$$
\boldsymbol{v}(\boldsymbol{x})=A \boldsymbol{x}+\boldsymbol{v}^{G o}(\boldsymbol{x})+\boldsymbol{v}^{N A}(\boldsymbol{x}) \quad \text { with } \quad \boldsymbol{v}^{N A}(\boldsymbol{x})=\boldsymbol{D}^{N A} \cdot \boldsymbol{x} .
$$

$\boldsymbol{v}^{G o}$ is the velocity field of [6] (recalled in Appendix B) and satisfies $\boldsymbol{v}^{G o}=\boldsymbol{D}^{A} \cdot \boldsymbol{x}$ on $\partial \Omega$ where $\boldsymbol{D}=\dot{\boldsymbol{E}}-A \boldsymbol{i}$ can be split into an axisymmetric part $\boldsymbol{D}^{A}$ and a non-axisymmetric part $\boldsymbol{D}^{N A}$ as in (19). The variational principle (2), when used with this compressible trial velocity field, gives:

$$
\Phi(\dot{\boldsymbol{E}}) \leq \inf _{A} \frac{1}{|\Omega|} \int_{\Omega-\omega} \sigma_{0} \int_{q_{1} f_{b}}^{1} \sqrt{\frac{4 A^{2}}{y^{2}}+\frac{\dot{\varepsilon}_{e q}^{2}}{q_{3}}} d y d \Omega, \quad \dot{\varepsilon}=\varepsilon(\boldsymbol{v}),
$$


and after use of the Cauchy-Schwarz inequality:

$$
\Phi(\dot{\boldsymbol{E}}) \leq \inf _{A} \sigma_{0} \int_{q_{1} f_{b}}^{1} \int_{\lambda_{1}}^{\lambda_{2}} \sqrt{J^{2}(\lambda) \frac{4 A^{2}}{y^{2}}+\frac{J(\lambda)}{q_{3}} \int_{\phi=0}^{2 \pi} \int_{\beta=0}^{\pi} \dot{\varepsilon}_{e q}^{2} J_{\lambda \beta \phi} d \beta d \phi} d \lambda d y .
$$

The last term can be computed following the same lines as in the above section:

$$
\Phi(\dot{\boldsymbol{E}}) \leq \inf _{A} \sigma_{0} \int_{q_{1} f_{b}}^{1} \int_{\lambda_{1}}^{\lambda_{2}} \sqrt{J^{2}(\lambda) \frac{4 A^{2}}{y^{2}}+\frac{J(\lambda)}{q_{3}} \boldsymbol{D}: \boldsymbol{Q}(\lambda): \boldsymbol{D}} d \lambda d y .
$$

3.3. Compressible GTN matrix: upper bound for the assemblage of randomly oriented hollow ellipsoids

The velocity field (23) satisfies boundary conditions of uniform deformation on the outer surface of the hollow ellipsoid $\boldsymbol{v}=\dot{\boldsymbol{E}} \cdot \boldsymbol{x}$ on $\partial \Omega$. These particular boundary conditions allow for the extension of this field into a velocity field which is continuous over the entire assemblage of hollow ellipsoids (it would not be the case with velocity fields satisfying other boundary conditions). Using the extended velocity field into the variational principle (2) leads to an upper bound for the assemblage ( $\mathcal{R}$ denotes a dependence on the orientation of the quantity under consideration and $\oint$ is the average over all possible orientations):

$$
\Phi(\dot{\boldsymbol{E}}) \leq \oint \sigma_{0} \inf _{A(\mathcal{R})} \int_{q_{1} f_{b}}^{1} \int_{\lambda_{1}}^{\lambda_{2}} \sqrt{J^{2}(\lambda) \frac{4 A^{2}(\mathcal{R})}{y^{2}}+\frac{J(\lambda)}{q_{3}} \boldsymbol{D}(\boldsymbol{\mathcal { R }}): \boldsymbol{Q}(\boldsymbol{\mathcal { R }}, \lambda): \boldsymbol{D}(\boldsymbol{\mathcal { R }})} d \lambda d y d \boldsymbol{\mathcal { R }} .
$$

The dilatation rate $A$ varies from one ellipsoid to another. But having in mind that the geometry of the assemblage is not known in detail and that we do not wish to keep track of a heterogenous dilatation-rate field, attention is restricted to a single $A$, independent of $\mathcal{R}$, for all the ellipsoids and uniform throughout the assemblage (this choice preserves the upper bound character of (26)). Therefore:

$$
\Phi(\dot{\boldsymbol{E}}) \leq \sigma_{0} \oint_{\inf _{A}} \int_{q_{1} f_{b}}^{1} \int_{\lambda_{1}}^{\lambda_{2}} \sqrt{J^{2}(\lambda) \frac{4 A^{2}}{y^{2}}+\frac{J(\lambda)}{q_{3}} \boldsymbol{D}: \boldsymbol{Q}(\boldsymbol{\mathcal { R }}, \lambda): \boldsymbol{D}} d \lambda d y d \boldsymbol{\mathcal { R }} .
$$

Then, using the fact the sum of infima is bounded from above by the infimum of the sum, one gets that:

$$
\Phi(\dot{\boldsymbol{E}}) \leq \sigma_{0} \inf _{A} \oint \int_{q_{1} f_{b}}^{1} \int_{\lambda_{1}}^{\lambda_{2}} \sqrt{J^{2}(\lambda) \frac{4 A^{2}}{y^{2}}+\frac{J(\lambda)}{q_{3}} \boldsymbol{D}: \boldsymbol{Q}(\boldsymbol{\mathcal { R }}, \lambda): \boldsymbol{D}} d \lambda d y d \boldsymbol{\mathcal { R }}
$$

and making use of the Cauchy-Schwarz inequality the following upper bound is obtained:

$$
\Phi(\dot{\boldsymbol{E}}) \leq \sigma_{0} \inf _{A} \int_{q_{1} f_{b}}^{1} \int_{\lambda_{1}}^{\lambda_{2}} \sqrt{J^{2}(\lambda) \frac{4 A^{2}}{y^{2}}+\frac{J(\lambda)}{q_{3}} \oint \boldsymbol{D}: \boldsymbol{Q}(\boldsymbol{\mathcal { R }}, \lambda): \boldsymbol{D} d \boldsymbol{\mathcal { R }}} d \lambda d y .
$$

Following Gatt et al [5] we note that:

$$
\oint \boldsymbol{D}: \boldsymbol{Q}(\boldsymbol{\mathcal { R }}, \lambda): \boldsymbol{D} d \boldsymbol{\mathcal { R }}=3 Q_{J} D_{m}^{2}+\frac{3}{10} Q_{K} D_{e q}^{2} \quad \text { with } \quad Q_{J}=\boldsymbol{Q}:: \boldsymbol{J} \quad \text { and } \quad Q_{K}=\boldsymbol{Q}:: \boldsymbol{K},
$$

where $\boldsymbol{J}$ and $\boldsymbol{K}$ are the usual fourth-order projectors over purely isotropic and purely deviatoric symmetric second-order tensors. Finally, after a few algebraic operations, the following upper bound is obtained:

$$
\Phi(\dot{\boldsymbol{E}}) \leq \sigma_{0} \inf _{A} \int_{q_{1} f_{b}}^{1} \int_{\lambda_{1}}^{\lambda_{2}} \sqrt{J^{2}(\lambda) \frac{4 A^{2}}{y^{2}}+\frac{J(\lambda)}{q_{3}}\left(3 Q_{J}(\lambda)\left(\dot{E}_{m}-A\right)^{2}+J(\lambda) \dot{E}_{e q}^{2}\right)} d \lambda d y,
$$

with

$Q_{J}(\lambda)=\frac{4}{9} \frac{b \pi}{|\Omega|}\left(b^{2}\left(-1+6 R \tilde{\alpha}+3 Z_{2} \tilde{\alpha}\right)^{2}+2 a^{2}\left(1-6 Z_{2} \tilde{\alpha}+12 R^{2} \tilde{\alpha}^{2}+12 Z^{2} \tilde{\alpha}^{2}+9 Z_{2}^{2} \tilde{\alpha}^{2}+6 Z \tilde{\alpha}\left(1+2 R \tilde{\alpha}-3 Z_{2} \tilde{\alpha}\right)\right)\right)$ 
where all quantities $a, b, R, Z, \tilde{\alpha} \ldots$ are defined in Appendix B and depend on $\lambda$.

\subsection{Compressible GTN matrix: Gurson-like estimate for the assemblage of randomly oriented hollow} ellipsoids

The final expression (28) is a rigorous upper bound for any assemblage of randomly oriented self-similar hollow ellipsoids. It involves a minimization over $A$ and a double integral which have to be evaluated numerically. In practice it can be useful to have an estimate (hopefully accurate) for the effective potential $\Phi$ or for the corresponding yield function in the space of macroscopic stresses.

For this purpose, the study of a single hollow ellipsoid with compressible matrix is reconsidered starting from equation (24). Using a few changes of variables and approximations almost identical to that initially proposed by Gologanu et al [6] and detailed in the Appendix C, we arrive at the following estimate for the effective potential of a single hollow ellipsoid:

$$
\Phi(\dot{\boldsymbol{E}}) \approx \inf _{A} \sigma_{0}^{\prime} \int_{q_{1} f_{b}}^{1} \int_{y_{2} / y_{1}}^{1} \sqrt{\frac{4 A^{2}}{y^{2}}+\boldsymbol{D}: \boldsymbol{S}(z): \boldsymbol{D}} d y d z,
$$

where $\sigma_{0}^{\prime}$, the $y_{i}$ 's and the fourth-order tensor $\boldsymbol{S}$ are specified in Appendix C. Then, averaging this estimate over all possible orientations and using the Cauchy-Schwarz inequality as in section 3.3, the following estimate is obtained:

$$
\Phi(\dot{\boldsymbol{E}}) \approx \inf _{A} \sigma_{0}(g+1) \int_{q_{1} f_{b}}^{1} \int_{\tilde{f}}^{1} \sqrt{\frac{4 A^{2}}{y^{2}}+\frac{\left(\dot{E}_{m}-A\right)^{2}}{z^{2}} \frac{\tilde{a}^{2}}{q_{3}}+\left(\dot{E}_{m}-A\right)^{2} \frac{\tilde{b}^{2}}{q_{3}}+\frac{\left(\dot{E}_{e q}\right)^{2}}{q_{3}}} d z d y,
$$

where $\tilde{a}, \tilde{b}, \tilde{f}$ and $g$ are specified in Appendix C. When the large voids are spherical, it follows from the detailed expressions given in Appendix $\mathrm{C}$ that $\tilde{a}=2, \tilde{b}=0$ and $g=0$. The potential (30) reduces to the upper bound (8).

Again, the form (30) is not very convenient for practical purposes and an analytical estimate for the associated yield function is searched for in the general form of the Gurson yield function. Under pure deviatoric macroscopic stress the prediction of the model (30) is

$$
\Sigma_{m}=0, \Sigma_{e q}=\tilde{\sigma}_{0} \text { with } \tilde{\sigma}_{0}=\sigma_{0}\left(1-f_{e}\right)\left(1-q_{1} f_{b}\right) / \sqrt{q_{3}},
$$

irrespective of the aspect ratio of the ellipsoid. This prediction is clearly an upper bound for the actual strength of the bi-porous material under pure shear. It is possible to improve on this upper bound by making use of the variational bound (15). As in section 2.4, the effective moduli $\widetilde{k}_{1}$ and $\widetilde{\mu}_{1}$ can be evaluated by a linear scheme appropriate for the microstructure under consideration (for randomly oriented confocal ellipsoids, the Ponte-Castañeda-Willis [16] bound seems to be most appropriate). The resulting expression is too complicated to be given in closed form, but its implementation is straightforward.

In order to determine the flow stress under hydrostatic loading conditions, the following expression is proposed for $A$ :

$$
A=\bar{A} \equiv \frac{q_{1} f_{b}}{\tilde{f}+q_{1} f_{b}} \dot{E}_{m} .
$$

This choice for $A$ leads under hydrostatic stress to:

$$
\Sigma_{e q}=0, \Sigma_{m}=\tilde{p} \text { with } \tilde{p}=\frac{1}{3} \sigma_{0}(g+1) I
$$

where $I$ is an integral whose detailed expression is given in Appendix A with

$$
f_{t}=\tilde{f}, \quad f_{s}=q_{1} f_{b}, \quad \tilde{P}=2\left|\frac{q_{1} f_{b}}{\tilde{f}+q_{1} f_{b}}\right|, \quad \tilde{Q}=\frac{1}{\sqrt{q_{3}}}\left|\frac{\tilde{f} \tilde{a}}{\tilde{f}+q_{1} f_{b}}\right| \quad \text { and } \quad \tilde{R}=\frac{1}{\sqrt{q_{3}}}\left|\frac{\tilde{f} \tilde{f}}{\tilde{f}+q_{1} f_{b}}\right| .
$$


The approximate yield function can be written as in (14) using the above expressions for $\tilde{\sigma}_{0}$ and $\tilde{p}$.

A comparison between the corresponding estimate and the upper bound (28) is shown in Figure 6 . The aspect ratio of the voids is defined as $w=a_{1} / b_{1}$ ( $w \leq 1$ corresponds to oblate voids). The agreement between the bound and the estimate is very good.

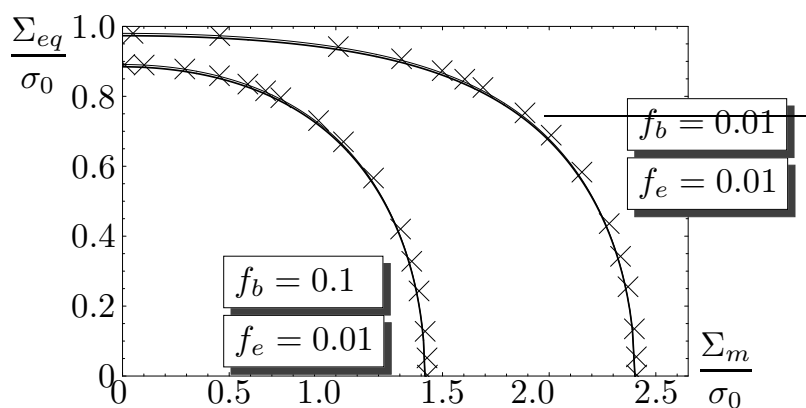

(a)

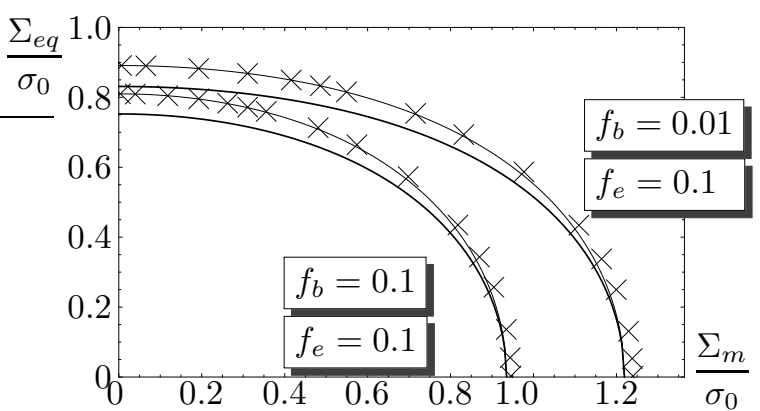

(b)

Figure 6. Effective yield surface of a Gurson material $\left(q_{1}=q_{3}=1\right)$ containing randomly oriented oblate ellipsoidal voids $(w=1 / 5)$. Comparison between the upper bound (28) (crosses), the estimate (14) (solid line) with (31) and (33) and the estimate (14) (thick solid line) with (33) and (15) where $\widetilde{\mu}_{1}$ is estimated with the PCW bound [16]. 


\section{Appendix A. Detailed expression of the integral $I$}

$$
I=\int_{f_{s}}^{1} \int_{f_{t}}^{1} \sqrt{\frac{\tilde{P}^{2}}{y^{2}}+\frac{\tilde{Q}^{2}}{z^{2}}+\tilde{R}^{2}} d z d y
$$

Introducing the notations:

$$
\begin{gathered}
\tilde{B}_{1}=\sqrt{\tilde{P}^{2}+\tilde{Q}^{2}+\tilde{R}^{2}}, \quad \tilde{B}_{2}=\sqrt{\tilde{P}^{2}+f_{s}^{2}\left(\tilde{Q}^{2}+\tilde{R}^{2}\right)}, \quad \tilde{B}_{3}=\sqrt{\tilde{Q}^{2}+f_{t}^{2}\left(\tilde{P}^{2}+\tilde{R}^{2}\right)} \\
\tilde{B}_{4}=\sqrt{f_{s}^{2} \tilde{Q}^{2}+f_{t}^{2}\left(\tilde{P}^{2}+f_{s}^{2} \tilde{R}^{2}\right)}, \quad \tilde{B}_{5}=\tilde{P} \tilde{Q} \tilde{R}, \quad \tilde{A}_{i}=\left(\tilde{P}^{2} \tilde{Q}^{2}-\tilde{R}^{2} \tilde{B}_{i}^{2}\right), \quad \tilde{C}_{i}=2 \tilde{B}_{5} \tilde{B}_{i}, \quad i=1,4, \\
K=\tilde{A}_{1} \tilde{A}_{4}-\tilde{C}_{1} \tilde{C}_{4}, \quad L=\tilde{A}_{1} \tilde{C}_{4}-\tilde{C}_{1} \tilde{A}_{4}, \quad M=\tilde{A}_{2} \tilde{A}_{3}-\tilde{C}_{2} \tilde{C}_{3}, \quad N=\tilde{A}_{2} \tilde{C}_{3}+\tilde{A}_{3} \tilde{C}_{2},
\end{gathered}
$$

an analytical expression for $I$ is obtained:

$$
\begin{aligned}
I= & \tilde{B}_{1}-\tilde{B}_{2}-\tilde{B}_{3}+\tilde{B}_{4}+\frac{\tilde{P} \tilde{Q}}{2 \tilde{R}} \arcsin \left(\frac{L M-N K}{M^{2}+N^{2}}\right)+ \\
& +\ln \left[\left(\frac{\tilde{Q}+\tilde{B}_{3}}{f_{t}\left(\tilde{Q}+\tilde{B}_{1}\right)}\right)^{\tilde{Q}}\left(\frac{f_{s}\left(f_{t} \tilde{P}+\tilde{B}_{3}\right)}{f_{t} \tilde{P}+\tilde{B}_{4}}\right)^{\left(\tilde{P} f_{t}\right)}\left(\frac{\tilde{P}+\tilde{B}_{2}}{f_{s}\left(\tilde{P}+\tilde{B}_{1}\right)}\right)^{\tilde{P}}\left(\frac{f_{t}\left(f_{s} \tilde{Q}+\tilde{B}_{2}\right)}{f_{s} \tilde{Q}+\tilde{B}_{4}}\right)^{\left(\tilde{Q} f_{s}\right)}\right] .
\end{aligned}
$$

\section{Appendix B. The velocity field (20) and related results}

The detailed construction of the velocity field used in the variational analysis on a single hollow ellipsoid is given here. The notations are essentially those of Gologanu et al [6]. The distance between the two foci of the confocal ellipsoids is $c=\sqrt{b_{1}^{2}-a_{1}^{2}}=\sqrt{b_{2}^{2}-a_{2}^{2}}$ and the hollow ellipsoidal region occupied by the matrix can be described in cylindrical coordinates $(\rho, \phi, z)$ by:

$$
\rho=c \cosh \lambda \sin \beta, \quad \phi=\phi, \quad z=c \sinh \lambda \cos \beta, \quad \lambda \in\left[\lambda_{1}, \lambda_{2}\right], \quad \beta \in[0, \pi], \quad \phi \in[0,2 \pi] .
$$

The inner and outer ellipsoids correspond to $\lambda=\lambda_{1}$ and $\lambda=\lambda_{2}$ respectively. The surfaces $\lambda=$ constant are confocal ellipsoids with minor and major semi-axes given by:

$$
a=c \sinh \lambda \quad \text { and } \quad b=c \cosh \lambda .
$$

Additional relations are useful:

$$
f_{e}=\frac{a_{1} b_{1}^{2}}{a_{2} b_{2}^{2}}, \quad e_{2}=\frac{c}{b_{2}}, \quad e_{1}=\frac{c}{b_{1}} \quad \text { and } \quad e=\frac{c}{b} .
$$

The incompressible velocity field proposed by Gologanu et al [6] reads as:

$$
\boldsymbol{v}^{G o}=\mathcal{A}(\dot{\boldsymbol{E}}) \boldsymbol{v}^{(\mathcal{A})}+\mathcal{B}(\dot{\boldsymbol{E}}) \boldsymbol{v}^{(\mathcal{B})} \quad \text { with } \quad \boldsymbol{v}^{(\mathcal{A})}=R(\lambda) \rho \boldsymbol{e}_{\rho}+Z(\lambda) z \boldsymbol{e}_{z}, \quad \boldsymbol{v}^{(\mathcal{B})}=-\frac{x}{2} \boldsymbol{e}_{x}-\frac{y}{2} \boldsymbol{e}_{y}+z \boldsymbol{e}_{z},
$$

with

$$
\begin{aligned}
& R(\lambda)=-a c / b^{2}+\arcsin (c / b) \quad \text { and } \quad Z(\lambda)=2 c / a-2 \arcsin (c / b), \\
& \mathcal{A}(\dot{\boldsymbol{E}})=3 \dot{E}_{m} /\left(2 R_{2}+Z_{2}\right), \quad \mathcal{B}(\dot{\boldsymbol{E}})=\dot{E}_{z z}-\mathcal{A} Z_{2}, R_{2}=R\left(\lambda_{2}\right), Z_{2}=Z\left(\lambda_{2}\right) .
\end{aligned}
$$


Note that $\boldsymbol{v}^{G o}=\dot{\boldsymbol{E}}^{A} \cdot \boldsymbol{x}$ on $\partial \Omega$.

When the second-order tensor $\dot{\boldsymbol{E}}$ is stored in vector form as:

$$
\dot{\boldsymbol{E}}=\left(\dot{E}_{x x}, \dot{E}_{y y}, \dot{E}_{z z}, \sqrt{2} \dot{E}_{x y}, \sqrt{2} \dot{E}_{x z}, \sqrt{2} \dot{E}_{y z}\right),
$$

the fourth-order tensor $\boldsymbol{Q}$, used in section 3, reads in matrix form as:

$$
\begin{aligned}
& \boldsymbol{Q}(\lambda)=\boldsymbol{T}^{T} \cdot \frac{2 \pi b}{|\Omega|}\left(\begin{array}{cc}
I_{1} & I_{2} \\
I_{2} & I_{3}
\end{array}\right) \cdot \boldsymbol{T}+J(\lambda) \boldsymbol{Q}^{N A}, \quad I_{1}=\frac{8}{9}\left(3 b^{2} R^{2}+2 a^{2}\left(R^{2}+R Z+Z^{2}\right)\right), \\
& I_{2}=\frac{4}{3}\left(-b^{2} R+a^{2} Z\right), \quad I_{3}=\frac{4 a^{2}}{3}+\frac{2 b^{2}}{3}, \quad \tilde{\alpha}=\frac{1}{2 R_{2}+Z_{2}}=\frac{a_{2} b_{2}^{2}}{2 c^{3}} .
\end{aligned}
$$

$\boldsymbol{T}$ is a $2 \times 6$ matrix and $\boldsymbol{Q}^{N A}$ is a symmetric $6 \times 6$ matrix whose non-zero entries are:

$$
\begin{aligned}
& T_{11}=T_{12}=T_{13}=\tilde{\alpha}, T_{21}=T_{22}=-\tilde{\alpha} Z_{2}, T_{23}=1-\tilde{\alpha} Z_{2}, \\
& Q_{11}^{N A}=Q_{22}^{N A}=-Q_{12}^{N A}=\frac{1}{3}, Q_{44}^{N A}=Q_{55}^{N A}=Q_{66}^{N A}=\frac{2}{3} .
\end{aligned}
$$

Remark: The nontrivial part of the Gurson velocity field (3) is the solution of the elasticity problem (under the constraint of incompressibility) of a hollow sphere under hydrostatic loading. Following up on this observation, a quite natural choice for a sensible velocity field in the problem of the hollow ellipsoid would be the exact solution of an incompressible elasticity problem posed on the hollow ellipsoid. Although very few exact results are available for such a geometry, the exact solution of the elasticity problem corresponding to a hydrostatic deformation of the inner ellipsoid and to a uniform deformation $\boldsymbol{D}$ on the outer ellipsoid is known (Milton [11]), with

$$
\begin{gathered}
\boldsymbol{D}=D_{x x}\left(\boldsymbol{e}_{x} \otimes \boldsymbol{e}_{x}+\boldsymbol{e}_{y} \otimes \boldsymbol{e}_{y}\right)+D_{z z} \boldsymbol{e}_{z} \otimes \boldsymbol{e}_{z}, \quad D_{x x}=-\frac{1}{3}+\alpha\left(e_{1}\right)-f_{e} \alpha\left(e_{2}\right), \quad D_{z z}=-2 D_{x x}-f_{e}, \\
\alpha(e)=-\frac{1-e^{2}}{2 e^{2}}+\frac{\sqrt{1-e^{2}}}{2 e^{3}} \arcsin (e) .
\end{gathered}
$$

Interestingly, this solution coincides with the field $\boldsymbol{v}^{G o}$ when $\dot{\boldsymbol{E}}=\boldsymbol{D}$. Therefore the velocity field of Gologanu et al [6] illustrates once again the interest of using solutions of elasticity problems as trial fields for ideally plastic problems (as first suggested by Gărăjeu [4] and further explored by Monchiet et al [12]).

\section{Appendix C. Derivation of the estimates (29) and (30)}

The starting point of this derivation is the relation (24). The integral

$$
\int_{\phi=0}^{2 \pi} \int_{\beta=0}^{\pi} \varepsilon_{e q}^{2}\left(\boldsymbol{v}^{G o}\right) J_{\lambda \beta \phi} d \beta d \phi
$$

is evaluated following [6] by replacing $\cos ^{2} \beta$ by $1 / 3$. Then, adopting the same sequence of changes of variables as [6] and the same approximations on $\varepsilon_{e q}^{2}\left(\boldsymbol{v}^{G o}\right)$ in $(24)$ leads to an estimate for $\Phi(\dot{\boldsymbol{E}})$. The sequence of changes of variables reads as:

$$
\tilde{x}=c^{3} /\left(a b^{2}\right)=\frac{1}{\sinh \lambda \cosh ^{2} \lambda}, \quad \text { then } \quad \tilde{y}=\frac{\chi \tilde{x}}{\tilde{x}+3 \chi / 4} \quad \text { with } \quad \chi=\sqrt{\pi^{2}+32 / 3} \quad \text { and finally } \quad z=\frac{y_{2}}{\tilde{y}} .
$$


The following estimate is obtained

$$
\Phi(\dot{\boldsymbol{E}}) \approx \inf _{A} \sigma_{0}^{\prime} \int_{q_{1} f_{b}}^{1} \int_{y_{2} / y_{1}}^{1} \sqrt{\frac{4 A^{2}}{y^{2}}+\frac{\mathcal{A}^{\prime 2}(\boldsymbol{D}) y_{2}^{2}}{q_{3} z^{2}}+\frac{\mathcal{B}^{\prime 2}(\boldsymbol{D})}{q_{3}}+\frac{\left(\dot{E}_{e q}^{N A}\right)^{2}}{q_{3}}} d z d y
$$

with:

$$
\sigma_{0}^{\prime}=\frac{16 \pi c^{3} \sigma_{0}}{9|\Omega| y_{2}}, \quad y_{2}=\tilde{y}\left(\tilde{x}\left(\lambda_{2}\right)\right), \quad y_{1}=\tilde{y}\left(\tilde{x}\left(\lambda_{1}\right)\right), \quad \mathcal{A}^{\prime}=\mathcal{A}(\boldsymbol{D}) \bar{F}+\mathcal{B}(\boldsymbol{D}) \bar{G}, \quad \mathcal{B}^{\prime}=\mathcal{B}(\boldsymbol{D}) \bar{H},
$$

and $\bar{F}, \bar{G}$ and $\bar{H}$ are constants which can be found in [6]. The term

$$
\frac{\mathcal{A}^{\prime 2}(\boldsymbol{D}) y_{2}^{2}}{z^{2}}+\mathcal{B}^{\prime 2}(\boldsymbol{D})
$$

can be developped by considering that $\bar{H}^{2}+\bar{G}^{2} y^{2} \approx 1$. The result reads as:

$$
\Phi(\dot{\boldsymbol{E}}) \approx \inf _{A} \sigma_{0}^{\prime} \int_{q_{1} f_{b}}^{1} \int_{y_{2} / y_{1}}^{1} \sqrt{\frac{4 A^{2}}{y^{2}}+\left[\bar{F}^{2} \mathcal{A}^{2}(\boldsymbol{D})+2 \bar{F} \bar{G} \mathcal{A}(\boldsymbol{D}) \mathcal{B}(\boldsymbol{D})\right] \frac{y_{2}^{2}}{q_{3} z^{2}}+\frac{\mathcal{B}^{2}(\boldsymbol{D})}{q_{3}}+\frac{\left(\dot{E}_{e q}^{N A}\right)^{2}}{q_{3}}} d z d y
$$

Then the term

$$
\left[\bar{F}^{2} \mathcal{A}^{2}(\boldsymbol{D})+2 \bar{F} \bar{G} \mathcal{A}(\boldsymbol{D}) \mathcal{B}(\boldsymbol{D})\right] \frac{y_{2}^{2}}{q_{3} z^{2}}+\frac{\mathcal{B}^{2}(\boldsymbol{D})}{q_{3}}+\frac{\left(\dot{E}_{e q}^{N A}\right)^{2}}{q_{3}},
$$

can easily be written as $\boldsymbol{D}: \boldsymbol{S}: \boldsymbol{D}$ where $\boldsymbol{S}$ is a fourth-order tensor.

The next step concerns randomly oriented ellipsoids. Following the same procedure as in section 3.3 and introducing the projections of $\boldsymbol{S}$ over $\boldsymbol{J}$ and $\boldsymbol{K}$, one obtains:

$$
\begin{aligned}
\Phi(\dot{\boldsymbol{E}}) & \approx \inf _{A} \sigma_{0}^{\prime} \int_{q_{1} f_{b}}^{1} \int_{y_{2} / y_{1}}^{1} \sqrt{\frac{4 A^{2}}{y^{2}}+\frac{\left(\dot{E}_{m}-A\right)^{2}}{z^{2}} \frac{\tilde{a}^{2}}{q_{3}}+\left(\dot{E}_{m}-A\right)^{2} \frac{\tilde{b}^{2}}{q_{3}}+\frac{\left(\dot{E}_{e q}\right)^{2}}{q_{3}}} d z d y \\
& \approx \inf _{A} \sigma_{0}(g+1) \int_{q_{1} f_{b}}^{1} \int_{\tilde{f}}^{1} \sqrt{\frac{4 A^{2}}{y^{2}}+\frac{\left(\dot{E}_{m}-A\right)^{2}}{z^{2}} \frac{\tilde{a}^{2}}{q_{3}}+\left(\dot{E}_{m}-A\right)^{2} \frac{\tilde{b}^{2}}{q_{3}}+\frac{\left(\dot{E}_{e q}\right)^{2}}{q_{3}}} d z d y
\end{aligned}
$$

with:

$$
\begin{gathered}
\tilde{f}=\frac{g+f_{e}}{g+1}, g=\frac{4 e_{2}^{3}}{3 \chi \sqrt{1-e_{2}^{2}}}, \tilde{a}^{2}=\frac{3}{\kappa^{2}(g+1)^{2}}\left(3-2 \eta+\frac{4 \eta Z_{2}}{\chi g}\right), \tilde{b}^{2}=\left(1-\frac{2}{\chi g} Z_{2}\right)^{2}, \\
Z_{2}=\frac{2 e_{2}}{\sqrt{1-e_{2}^{2}}}-2 \arcsin \left(e_{2}\right), \kappa=\left(\frac{2}{3}+\frac{g(1-f)(g+2 f+g f)}{3(g+1)^{2}(g+f)^{2} \log \frac{g+1}{g+f}}\right)^{-1}, \\
\eta=\frac{\kappa(1-f)(g+1)(g+f) \sinh \left(2 \kappa\left(\alpha_{2}-\alpha_{1}\right)\right)}{(g+1)^{2}+(g+f)^{2}+2(g+1)(g+f)\left(\kappa\left(\alpha_{2}-\alpha_{1}\right) \sinh \left(2 \kappa\left(\alpha_{2}-\alpha_{1}\right)\right)-\cosh \left(2 \kappa\left(\alpha_{2}-\alpha_{1}\right)\right)\right)},
\end{gathered}
$$

with $\alpha_{2}=\alpha\left(e_{2}\right), \alpha_{1}=\alpha\left(e_{1}\right)$, the function $\alpha(e)$ being defined in (B.5).

\section{Acknowledgements}

The authors are indebted to J.-C. Michel for providing them with the FEM code used in the numerical simulations shown in Figure 3. 


\section{References}

[1] N. Bilger, F. Auslender, M. Bornert, R. Masson, New bounds and estimates for porous media with rigid perfectly plastic matrix, Comptes Rendus Mécanique 330 (2002), 127-132.

[2] J.L. Chaboche, P. Suquet, J. Besson, Endommagement et changement d'échelle, in: M. Bornert, T. Bretheau, P. Gilormini (Eds.), Homogénéisation en mécanique des matériaux 2, Hermès Science Publications, Paris, 2001.

[3] F. Dherbey, F. Louchet, A. Mocellin, S. Leclercq, Elevated temperature creep of polycrystalline uranium dioxide: from microscopic mechanisms to macroscopic behaviour, Acta Mater. 50 (2002) 1495-1505.

[4] M. Gărăjeu, Contribution à l'étude du comportement non linéaire de milieux poreux avec ou sans renfort, PhD thesis, Aix-Marseille 2 University, 1995.

[5] J.M. Gatt, Y. Monerie, D. Laux, D. Baron, Elastic behavior of porous ceramics: application to nuclear fuel materials, J. Nuclear Materials 336 (2005) 145-155.

[6] M. Gologanu, J.B. Leblond, J. Devaux, Approximate models for ductile metals containing non-spherical voids-Case of axisymmetric oblate ellipsoidal cavities, ASME J. Engng. Mater. Technol. 116 (1994) 290-297.

[7] A.L. Gurson, Continuum theory of ductile rupture by void nucleation and growth: Part I - Yield criteria and flow rules for porous ductile media, J. Engng. Mater. Technol. 99 (1977) 2-15.

[8] J.B. Leblond, G. Perrin, P. Suquet, Exact Results and Approximate Models for Porous Viscoplastic Solids, Int. J. Plasticity 10 (1994) 213-235.

[9] J.B. Leblond, Mécanique de la Rupture fragile et ductile, Hermès, Paris, 2002.

[10] J.C. Michel, H. Moulinec, P. Suquet, Effective properties of composite materials with periodic microstructure: a computational approach, Comp. Meth. Appl. Mech. Engng. 172 (1999) 109-143.

[11] G.W. Milton, The Theory of Composites, Cambridge University Press, Cambridge, 2002.

[12] V. Monchiet, E. Charkaluk, D. Kondo, An improvement of Gurson-type models of porous materials by using Eshelby-like trial velocity fields, Comptes Rendus Mecanique 335 (2007) 32-41.

[13] G. Perrin, J.B. Leblond, Analytical study of a hollow sphere made of plastic porous material and subjected to hydrostatic tension. Application to some problems in ductile fracture of metals, Int. J. of Plasticity 6 (1990) 677-699.

[14] G. Perrin, Contribution à l'étude théorique et numérique de la rupture ductile des métaux, PhD thesis, Ecole Polytechnique, Palaiseau, 1992.

[15] G. Perrin, J.B. Leblond, Accelerated void growth in porous ductile solids containing two populations of cavities, Int. J. of Plasticity 16 (2000) 91-120.

[16] P. Ponte Castañeda, J.R. Willis, The effect of spatial distribution on the effective behavior of composite materials and cracked media, J. Mech. Phys. Solids, 43 (1995) 1919-1951.

[17] P. Suquet, On bounds for the overall potential of power law materials containing voids with arbitrary shape, Mech. Res. Comm. 19 (1992) 51-58.

[18] V. Tvergaard, Material Failure by Void Growth to Coalescence, in J.W. Hutchinson and T.Y. Wu (eds) Advances in Applied Mechanics 27 (1990) 83-151. 